\title{
Chapter 14 \\ Application of the Resource-Renewable Boiling Water Reactor for TRU Management and Long-Term Energy Supply
}

\author{
Tetsushi Hino, Masaya Ohtsuka, Renzo Takeda, Junichi Miwa, \\ and Kumiaki Moriya
}

\begin{abstract}
The RBWR (resource-renewable boiling water reactor) is an innovative BWR that has a capability to breed and burn trans-uranium elements (TRUs) using a multi-recycling process. The RBWR can be used as a long-term energy supply, and it reduces the negative environmental impact that TRUs cause as they are otherwise long-lived radioactive wastes. Various design concepts of the RBWR core have been proposed. The RBWR-AC is a break-even reactor and the RBWRTB and RBWR-TB2 are TRU burners. The RBWR-TB is designed to burn TRUs from the RBWR-TB itself and to burn almost all the TRUs by repeating their recycling. The RBWR-TB is assumed to be applied for a nuclear power phase-out scenario. The RBWR-TB2 is intended to burn TRUs from LWR spent fuels. The RBWR-TB2 is assumed to be applied for reducing the amount of TRUs to be managed in storage facilities. The RBWR cores achieve their TRU multi-recycling capability under the constraint that the void reactivity coefficient must be negative by introducing the parfait core concept. This chapter reviews details of the specific design and core characteristics of the RBWR.
\end{abstract}

Keywords Break-even • Burner • BWR • Multi-recycle $\bullet$ TRU $\bullet$ Void reactivity coefficient

\subsection{Introduction}

Nuclear-generated electrical power is one irreplaceable candidate energy source that responds to the needs for energy security and for reduction of greenhouse-gas emissions. However, there has also been growing concern that significant amounts

T. Hino $(\bowtie) \cdot$ M. Ohtsuka $\bullet$ R. Takeda $\bullet$ J. Miwa

Hitachi, Ltd., Hitachi Research Laboratory, 7-1-1, Omika-cho, Hitachi-shi

Ibaraki-ken 319-1292, Japan

e-mail: tetsushi.hino.kd@hitachi.com

K. Moriya

Hitachi-GE Nuclear Energy, Ltd., 3-1-1, Saiwai-cho, Hitachi-shi, Ibaraki-ken 317-0073, Japan

(C) The Author(s) 2015

K. Nakajima (ed.), Nuclear Back-end and Transmutation Technology for Waste

Disposal, DOI 10.1007/978-4-431-55111-9_14 
of trans-uranium elements (TRUs) are becoming long-lived radioactive wastes. If TRUs could be recycled as nuclear fuel, the benefits attained from nuclear power would increase as a long-term energy supply and the negative environmental impact of TRUs as radioactive wastes could be greatly reduced. For these purposes many types of innovative reactors, including the sodium-cooled fast reactor (SFR), have been proposed. The resource-renewable BWR (RBWR) has been proposed to achieve the same purposes using concepts based on proven BWR technologies and the BWR capability to control the neutron energy spectrum flexibly [1-3]. A major characteristic of the BWR is "boiling" in the core, which includes water that functions as both a moderator and a coolant. The neutron energy spectrum can be hardened by reducing the hydrogen-to-uranium ratio $(\mathrm{H} / \mathrm{U})$ using the two-phase flow and using the hexagonal tight fuel lattice, so that the transmutation of ${ }^{238} \mathrm{U}$ to fissile plutonium is promoted with increasing resonance absorption: this enables the multi-recycling process of both breeding and consuming TRUs. On the other hand, there is a tendency that the harder the neutron spectrum becomes in the TRU-loaded core, the more positive the void reactivity coefficient becomes. The void reactivity coefficient is one of the main safety parameters for light water reactors (LWRs) and must be negative. The RBWR achieves the TRU multi-recycling capability under the constraint of the negative void reactivity coefficient by introducing the parfait core concept [4].

This chapter reviews details of the specific design and core characteristics of the RBWR.

\subsection{RBWR System}

\subsubsection{Overview}

Figure 14.1 shows the reactor pressure vessel (RPV) of the RBWR. The common plant specifications of the RBWR and the latest commercial BWR, the ABWR, are listed in Table 14.1. The rated thermal power, electric power, diameter of the RPV, and core pressure are identical for both reactor plants. Figure 14.2 shows a horizontal cross-sectional view of the RBWR core configuration, which is composed of 720 hexagonal fuel bundles and 223 Y-type control rods. The axial configuration uses the parfait core concept in which an internal blanket of depleted uranium oxide is placed between the upper and lower fissile zones of the TRU oxides.

Various design concepts of the RBWR core have been proposed. Recent core designs have focused on TRU management. The RBWR-AC is the break-even reactor that can burn depleted uranium by using TRUs extracted from the spent fuel bundles of LWRs without decreasing the amount of TRUs. The RBWR-TB is the TRU burner that can fission almost all the TRUs, leaving only the minimum critical mass of TRUs, by repeating their recycling and collecting. The RBWR-TB2 is a modified version of the TRU burner. The RBWR-TB2 is designed to be able to burn 
Fig. 14.1 Reactor pressure vessel of the resourcerenewable boiling water reactor (RBWR) [3]

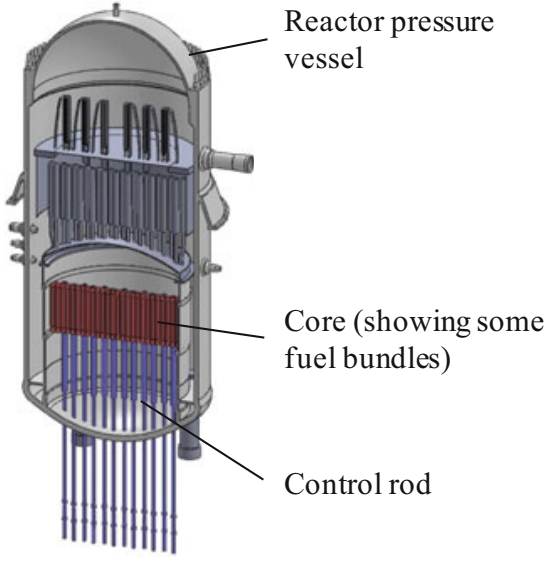

Table 14.1 Plant specifications [3]

\begin{tabular}{l|l|l}
\hline Item & RBWR & ABWR \\
\hline Thermal power (MWt) & 3,926 & 3,926 \\
\hline Electric power (MWe) & 1,356 & 1,356 \\
\hline RPV diameter (m) & 7.1 & 7.1 \\
\hline Core pressure (MPa) & 7.2 & 7.2 \\
\hline Number of fuel bundles & 720 & 872 \\
\hline Fuel lattice type & Hexagonal & Square \\
\hline Lattice pitch (mm) & 199 & 155 \\
\hline Number of control rods & 223 & 205 \\
\hline Control rod type & Y-type & Cross shape \\
\hline
\end{tabular}

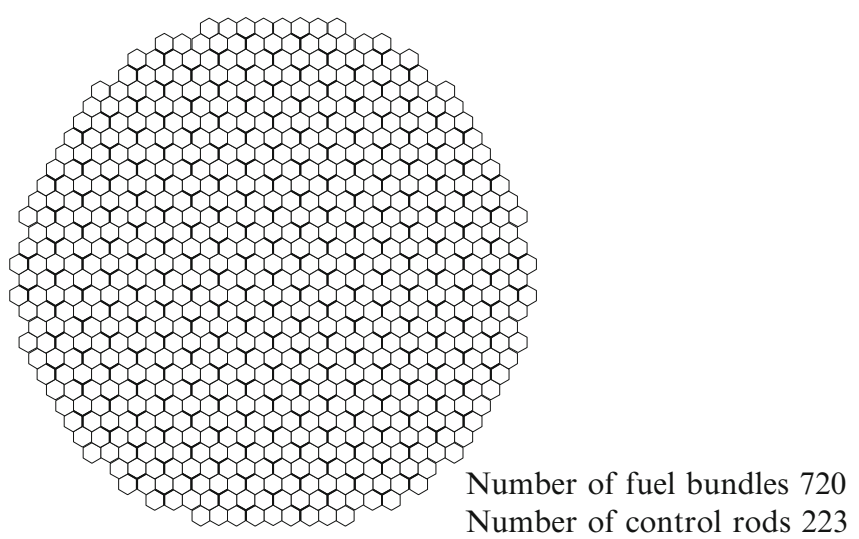

Fig. 14.2 Horizontal cross-sectional view of the RBWR core configuration [3] 


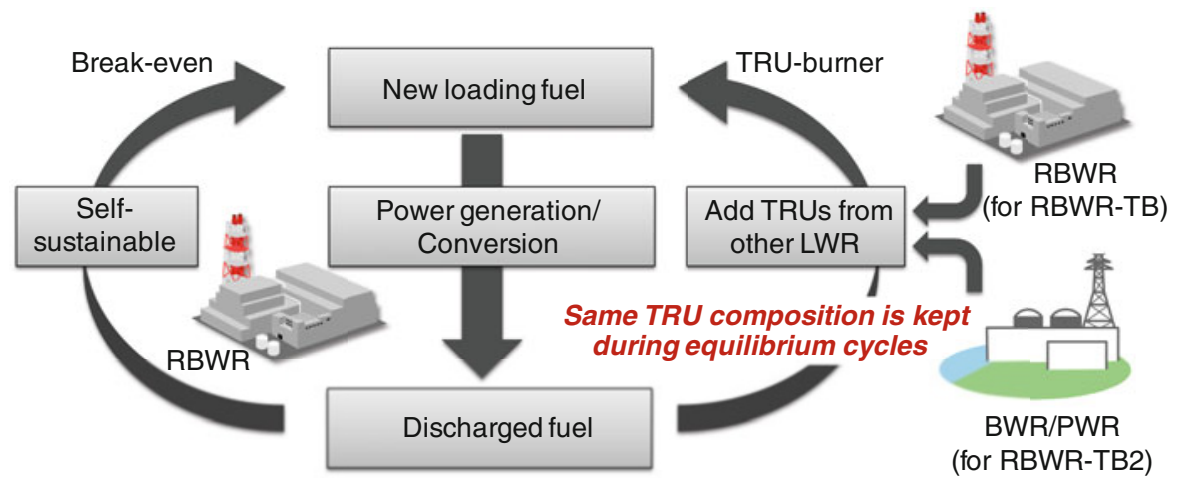

Fig. 14.3 Utilization concept of the RBWR-AC, -TB, and -TB2

TRUs from LWR spent fuels, whereas the RBWR-TB is designed as a burner for the TRUs from the RBWR-TB itself, assuming the RBWR-TB would be utilized when the TRU usefulness is exhausted and almost all should have been fissioned. Figure 14.3 shows the utilization concept of the RBWR-AC, -TB, and -TB2.

In core designs for the RBWR-AC, -TB, and -TB2, keeping charged TRU composition preserved at every operation cycle is mandatory. This criterion ensures the multi-recycling capability, fission, and recycling process of TRUs can be continued while maintaining the criticality and fulfilling the various operation constraints, such as sufficient reactor shutdown margin and negative void reactivity coefficient. As mentioned in the Introduction, the multi-recycling capability is achieved by hardening the neutron energy spectrum and promoting the transmutation of ${ }^{238} \mathrm{U}$ to fissile plutonium using the hexagonal tight fuel lattice, which has a $\mathrm{H} / \mathrm{U}$ less than that of the conventional BWR square fuel lattice. Figure 14.4 shows the relationship between the volume ratio of water to fuel and the breeding ratio in the RBWR-AC, -TB, -TB2, and the conventional BWR. Because the RBWR-AC and $-\mathrm{TB}$ need to continue operation cycles without feeding fissile materials other than those contained in the discharged fuel from themselves, the volume ratios of water to fuel are set lower than those of the RBWR-TB2 and the conventional BWR.

In the following sections, the core calculation method is described first, and then each type of RBWR is described.

\subsubsection{Core Calculation Method}

An outline of the calculation methods used for the core design is as follows. Group constants of 12 energy groups for the core neutronic calculation were evaluated for the horizontal cross section of the fuel bundle lattice by the Monte Carlo calculation code with 190 energy groups [5]. In the burn-up calculation, 45 actinides from 


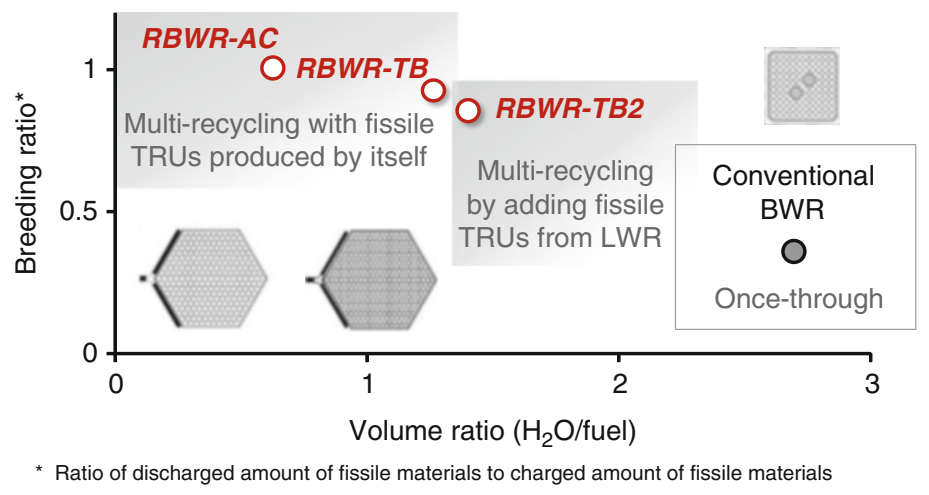

Fig. 14.4 Relationship between water to fuel volume ratio and fissile breeding ratio

${ }^{228}$ Th to ${ }^{253}$ Es and 84 fission products (83 nuclides treated explicitly and 1 lumped fission product) were treated. In the core neutronic calculation, the 12-energy group, three-dimensional neutron flux was obtained by solving the diffusion equation with 1 mesh for each fuel bundle in the horizontal direction and 34 meshes in the vertical direction.

In the thermal hydraulic calculation, the in-channel coolant flow rate, the two-phase flow pressure drop, and the axial void fraction distribution were calculated based on the power distribution obtained by the core neutronic calculation, so that the pressure drops between fuel bundles were balanced. The core neutronic calculation and the thermal hydraulic calculation were iterated until the power distribution and in-channel coolant flow distribution converged.

The void reactivity coefficient was evaluated by decreasing the core coolant flow rate to $95 \%$ of the rated flow and dividing the change of the neutron multiplication factor by the change of core averaged void fraction, from the respective values at the rated flow.

\subsubsection{RBWR-AC}

The axial fuel bundle configuration of the RBWR-AC is shown in Fig. 14.5. The axial configuration is the parfait core, where an internal blanket $(520 \mathrm{~mm})$ of depleted uranium oxide is placed between two fissile zones (upper, $280 \mathrm{~mm}$; lower, $193 \mathrm{~mm}$ ). The upper and lower blankets $(70$ and $280 \mathrm{~mm}$ ) are attached above and below the upper and lower fissile zones, respectively.

The neutron absorber zones are placed above and below the fuel zone (fissile and blanket) to increase the margin to maintain the negative void reactivity coefficient. The upper neutron absorber zone is composed of the neutron absorber rods placed between the plenums, which are connected to the fuel rods. The neutron absorber rods are filled with $\mathrm{B}_{4} \mathrm{C}$ pellets in a sealed tube with an outside diameter of $7.7 \mathrm{~mm}$. 
Fig. 14.5 Axial configuration of the RBWRAC fuel bundle [3]

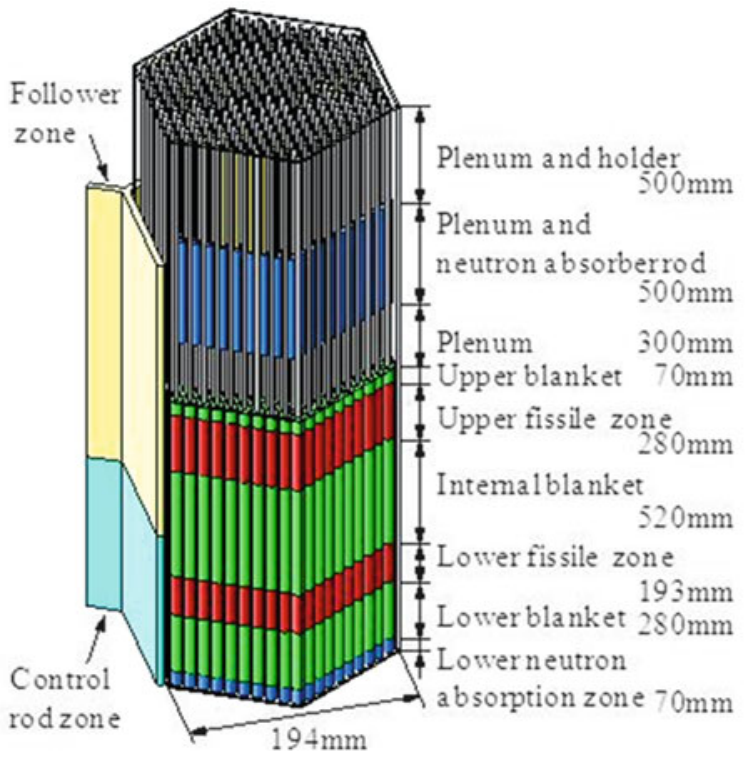

Each neutron absorber rod is attached to support rods fixed with the upper tie-plate of the fuel bundle. The neutron absorber rods are installed in a ratio of one per one fuel rod. Each neutron absorber rod is $500 \mathrm{~mm}$ long, and the distance between the upper end of the fuel zone and the lower end of the neutron absorber rod is $300 \mathrm{~mm}$. The lower neutron absorber zone is composed of $\mathrm{B}_{4} \mathrm{C}$ pellets filled in the fuel cladding. The length of the lower neutron absorber zone is $70 \mathrm{~mm}$.

Figure 14.6 shows a horizontal cross-sectional view of the configuration of the RBWR-AC fuel bundle and its fissile Pu enrichment distribution. The lattice pitches of the fuel bundles are $199.2 \mathrm{~mm}$ on the side with the control rod and $194.7 \mathrm{~mm}$ on the side without it. The channel box of the fuel bundle is hexagonal with an inner width of $189.1 \mathrm{~mm}$, and its wall thickness is $2.4 \mathrm{~mm}$. The control rod is $6.5 \mathrm{~mm}$ thick, and the gap between the rod outer surface and the channel box is $1.6 \mathrm{~mm}$ on each side; the gap between channel boxes on the side without the control rod is $0.8 \mathrm{~mm}$.

The fuel rod gap and pitch are 1.3 and $11.4 \mathrm{~mm}$, respectively. For the equilibrium core of the RBWR-AC, the bundle-averaged fissile plutonium enrichment is $15.7 \mathrm{wt} \%$ for the upper fissile zone (Fig. 14.6a) and $20.1 \mathrm{wt} \%$ for the lower fissile zone (Fig. 14.6b). Both the upper and lower fissile zones utilize five different fissile $\mathrm{Pu}$ enrichments.

The main core specifications and performance values of the RBWR-AC in the equilibrium core are shown in Table 14.2. The core coolant flow is $2.6 \times 10^{4} \mathrm{t} / \mathrm{h}$ at a subcooling temperature of $5 \mathrm{~K}$ at the entrance and has a steam quality of $35 \mathrm{w} / \mathrm{o}$ at the core exit. The void fraction of core coolant is about $30 \%$ at the bottom of the lower fissile zone because of heating in the lower blanket; it reaches $80 \%$ at the top of the core. A breeding ratio of 1.01 is achievable under a $45 \mathrm{GWd} / \mathrm{t}$ exposure 
14 Application of the Resource-Renewable Boiling Water Reactor for TRU...

a

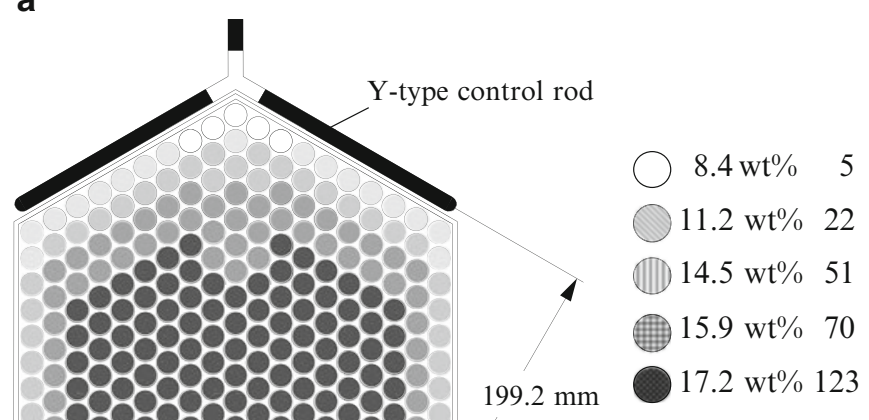

Average fissile Puenrichment $15.7 \mathrm{wt} \%$

Number of fuel rods

Fuel rod diameter

$10.1 \mathrm{~mm}$

$-194.7 \mathrm{~mm}$

Fuel rod gap

$1.3 \mathrm{~mm}$

Thickness of control rod

$6.5 \mathrm{~mm}$

Upper fissile zone

b
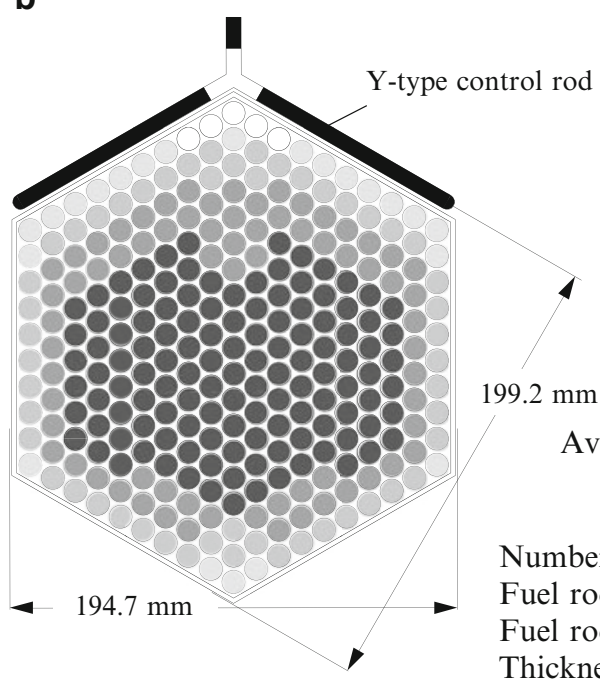

$199.2 \mathrm{~mm}$

$13.0 w t^{\%} \% \quad 5$

$15.8 w \mathrm{wt}^{\circ} \% 22$

(III) $19.1 \mathrm{wt}^{\circ} \% 51$

(1) $20.6 \mathrm{wt}^{\circ} \% \quad 70$

- $21.3 w \mathrm{wt}^{\circ} 123$

Average fissile Puenrichment $20.1 \mathrm{wt} \%$

Number of fuel rods

271

Fuel rod diameter

$10.1 \mathrm{~mm}$

Fuel rod gap

$1.3 \mathrm{~mm}$

Thickness of control rod $6.5 \mathrm{~mm}$

Lower fissile zone

Fig. 14.6 Horizontal cross-sectional view showing configuration of the RBWR-AC fuel bundle and fissile Pu enrichment distribution [3] 
Table 14.2 Core specifications and performance values [3]

\begin{tabular}{l|l|l|l}
\hline Item & RBWR-AC & RBWR-TB & RBWR-TB2 \\
\hline Core height $(\mathrm{mm})$ & 1,343 & 993 & 1,025 \\
\hline Fuel rod diameter $(\mathrm{mm})$ & 10.1 & 7.4 & 7.2 \\
\hline Fuel rod pitch $(\mathrm{mm})$ & 11.4 & 9.4 & 9.4 \\
\hline Fuel rod gap $(\mathrm{mm})$ & 1.3 & 2.0 & 2.2 \\
\hline Pellet diameter $(\mathrm{mm})$ & 8.7 & 6.1 & 6.0 \\
\hline Number of fuel rods & 271 & 397 & 397 \\
\hline Coolant flow rate $(\mathrm{t} / \mathrm{h})$ & $2.6 \times 10^{4}$ & $3.8 \times 10^{4}$ & $2.4 \times 10^{4}$ \\
\hline Core exit quality $(\%)$ & 35 & 21 & 36 \\
\hline Void fraction $(\%)$ & 53 & 42 & 56 \\
\hline Pressure drop $(\mathrm{MPa})$ & 0.14 & 0.19 & 0.06 \\
\hline HM inventory $(\mathrm{t})$ & 144 & 77 & 76 \\
\hline Puf/HM in fissile zone $(\mathrm{wt} \%)$ & $15.7 / 20.1$ & 13.9 & 25 \\
\hline Puf inventory $(\mathrm{t})$ & 9.0 & 4.5 & 8.3 \\
\hline Burn-up $(\mathrm{GWd} / \mathrm{t})$ & 45 & 55 & 65 \\
\hline MLHGR $(\mathrm{kW} / \mathrm{m})$ & 47 & 47 & 47 \\
\hline MCPR & 1.28 & 1.3 & 1.28 \\
\hline Void reactivity coefficient $(\Delta \mathrm{k} / \mathrm{k} / \%$ void) & $-2.4 \times 10^{-4}$ & $-2 \times 10^{-4}$ & $-4 \times 10^{-4}$ \\
\hline Breeding ratio & 1.01 & - & - \\
\hline TRU fission efficiency $(\%)$ & - & 51 & 45 \\
\hline
\end{tabular}

averaged with the upper, internal, and lower blankets. Here the breeding ratio is defined as the number of atoms of fissile plutonium left in the discharged fuel bundles per fissile plutonium loaded in the initial charged fuel bundles.

The loading pattern of the fuel bundles in the equilibrium core adopts zone loading with the reflective boundary condition of $60^{\circ}$ in the azimuthal direction. After the control rod scheduling is done, the radial power peaking factor is about 1.2 and the axial power peaking factor is about 1.8, including the blanket zones, which results in the minimum critical power ratio of 1.3 and the maximum linear heatgenerating rate of $47 \mathrm{~kW} / \mathrm{m}$.

The RBWR-AC has a void reactivity coefficient of $-2.4 \times 10^{-4} \Delta \mathrm{k} / \mathrm{k} / \%$ void, which is comparable with that of the current BWR, about $-7 \times 10^{-4} \Delta \mathrm{k} / \mathrm{k} / \%$ void.

\subsubsection{RBWR-TB}

The axial fuel bundle configuration of the RBWR-TB is shown in Fig. 14.7. The axial configuration is similar to that of the RBWR-AC, but the RBWR-TB does not have a lower blanket because breeding of fissile plutonium is not needed. Other blanket and fissile zones have different heights from those in the RBWR-AC to enable multi-recycling of TRUs under the different neutron energy spectrum from the RBWR-AC. The upper and internal blanket zones of depleted uranium oxides 
Fig. 14.7 Axial configuration of the RBWR-TB fuel bundle [3]

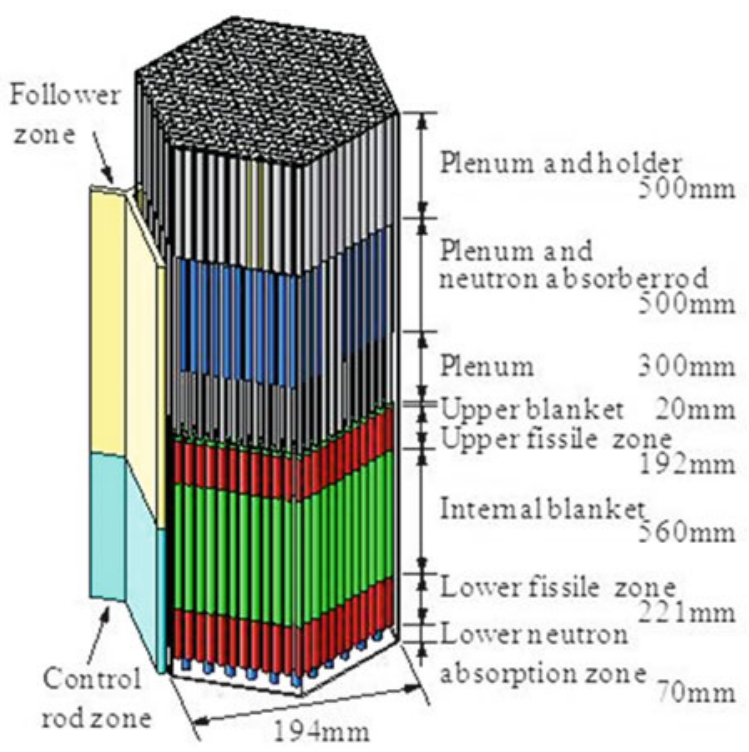

have heights of 20 and $560 \mathrm{~mm}$, respectively; the upper and lower fissile zones have heights of 192 and $221 \mathrm{~mm}$, respectively.

The RBWR-TB also utilizes the neutron absorber zones above and below the fuel zone. The upper neutron absorber zone has the same structure as that of the RBWR-AC. The number of neutron absorber rods in the lower neutron absorber zone is 91 , which was determined so as to keep the void reactivity coefficient negative.

Figure 14.8 shows the horizontal configuration of the RBWR-TB. The fuel bundle of the RBWR-TB is composed of the uniform fissile plutonium enrichment of $13.9 \mathrm{wt} \%$. The lattice pitches of the fuel bundles are $199.3 \mathrm{~mm}$ on the side with the control rod and $194.4 \mathrm{~mm}$ on the side without it. The channel box of the fuel bundle is hexagonal with an inner width of $189.6 \mathrm{~mm}$ and wall thickness of $2 \mathrm{~mm}$. The control rod is $7.5 \mathrm{~mm}$ thick, and the gap between the rod outer surface and the channel box is $1.6 \mathrm{~mm}$ on each side. The gap between channel boxes on the side without the control rod is $0.8 \mathrm{~mm}$. Geometries of the channel boxes and the control rods are slightly different from those of the RBWR-AC. However, because the center positions of the control rods are the same in the RBWR-AC and -TB and reactor internals fixed to the RPV, such as the core support plate, control rod guide tubes, etc., can be shared, their cores are easily exchanged with each other by changing the fuel bundles, control rods, and some attachments between the core support plate and fuel bundles.

Because the RBWR-TB equilibrium core has a shorter height than that of the RBWR-AC, the number of fuel rods of the RBWR-TB (397) is larger than that of the RBWR-AC (271) to keep the averaged linear heat-generating rate almost the same. 


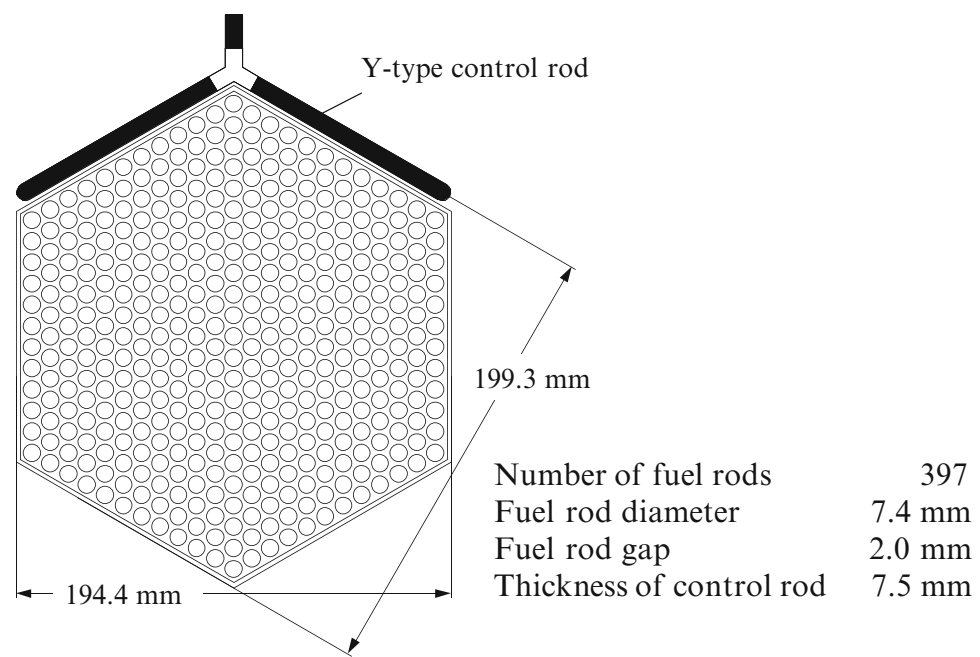

Fig. 14.8 Horizontal cross-sectional view showing configuration of the RBWR-TB fuel bundle [3]

The RBWR-TB aims at burning MAs by transmuting them into fissile isotopes using relatively low energy neutrons as well as by direct fissioning using relatively high energy neutrons. Both capture and fission reactions occur in a broad neutron energy range from thermal to fast. It is expected that the balance of these reactions at different neutron energies enables TRU burning while keeping the isotopic composition almost the same before and after burning, as mentioned in the next subsection.

The main core specifications and performance values of the RBWR-TB in the equilibrium core were shown earlier in Table 14.2. The core coolant flow is $3.8 \times 10^{4} \mathrm{t} / \mathrm{h}$ at a subcooling of $10 \mathrm{~K}$ at the entrance and has a steam quality of $21 \%$ at the core exit. The concept of the loading pattern of fuel bundles in the equilibrium core is the same as that of the RBWR-AC: it adopts zone loading and the reflective boundary condition of $60^{\circ}$ in the azimuthal direction. A maximum linear heat generation rate of $47 \mathrm{~kW} / \mathrm{m}$ and an MCPR of 1.3 after the control rod scheduling are achieved. The RBWR-TB has a void reactivity coefficient of $-2 \times 10^{-4} \Delta \mathrm{k} / \mathrm{k} / \%$ void.

The fission efficiency of TRUs in the RBWR-TB is $51 \%$. Here the fission efficiency is defined as the net decrease in TRUs divided by the total amount of fissioned actinides through the total fuel residence time in the core. This value indicates what amount of the TRUs can be used as fuel for generating electric power and is related to fissioning cost of the TRUs. As the fission efficiency of TRUs becomes higher, it is expected that the electricity-generating cost needed for burning the same amount of TRUs becomes smaller, if the other costs such as fuel fabrication cost are comparable. 
Fig. 14.9 Axial configuration of the RBWR-TB2 fuel bundle [3]

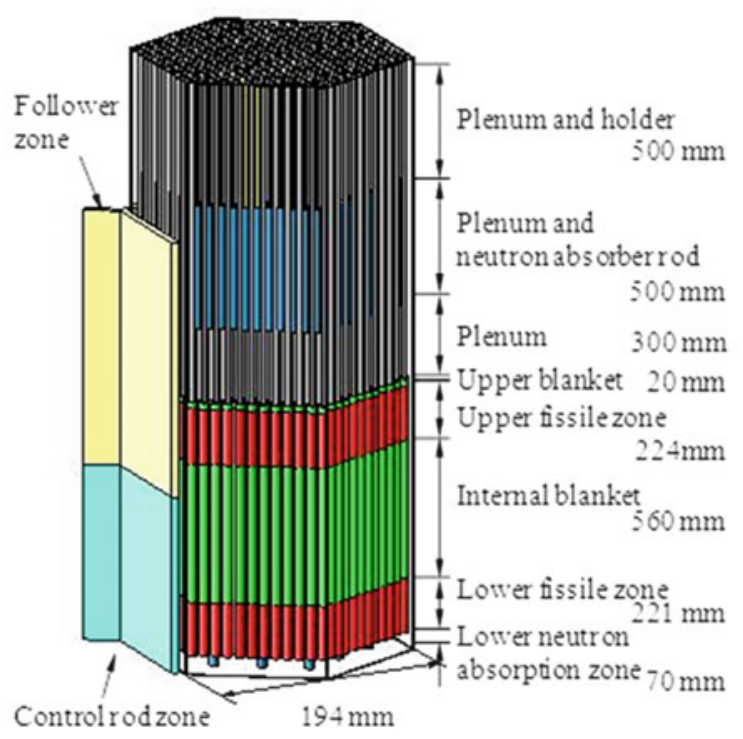

\subsubsection{RBWR-TB2}

The core concept of the RBWR-TB2 was initiated by an Electric Power Research Institute (ERRI)-organized team of three universities in the United States [6] to compare its core performance values with those of the ABR, which is the SFR having the same purpose [7]. Although the RBWR-TB is assumed to be utilized in the final stage of the nuclear power phase-out scenario, the RBWR-TB2 is assumed to be utilized to control the amount of TRUs during the period while LWRs are being operated as base load power sources.

The axial configuration of the RBWR-TB2 (Fig. 14.9) and it is similar to that of the RBWR-TB. The RBWR-TB2 also does not have a lower blanket because breeding of fissile plutonium is not needed. The upper and internal blanket zones of depleted uranium oxide have heights of 20 and $560 \mathrm{~mm}$, respectively; the upper and lower fissile zones have heights of 224 and $221 \mathrm{~mm}$, respectively. The RBWRTB2 also uses a lower neutron absorption zone in which the number of neutron absorber rods is 19. This number of the neutron absorber rods is sufficient to keep the void reactivity coefficient negative in the RBWR-TB2.

Figure 14.10 shows the horizontal configuration of the RBWR-TB2. The fuel bundle of the RBWR-TB2 is composed of the uniform fissile plutonium enrichment of $25 \mathrm{wt} \%$. As the RBWR-TB2 fuel includes TRUs from LWRs, the fissile plutonium enrichment becomes higher than that of the RBWR-TB, which uses TRUs from itself and other RBWR-TBs in the equilibrium core. Geometries of the channel box and the control rods are the same as those of the RBWR-TB. The lattice pitches of the fuel bundles are $199.3 \mathrm{~mm}$ on the side with the control rod and $194.4 \mathrm{~mm}$ on the side without it. The channel box of the fuel bundle is hexagonal 


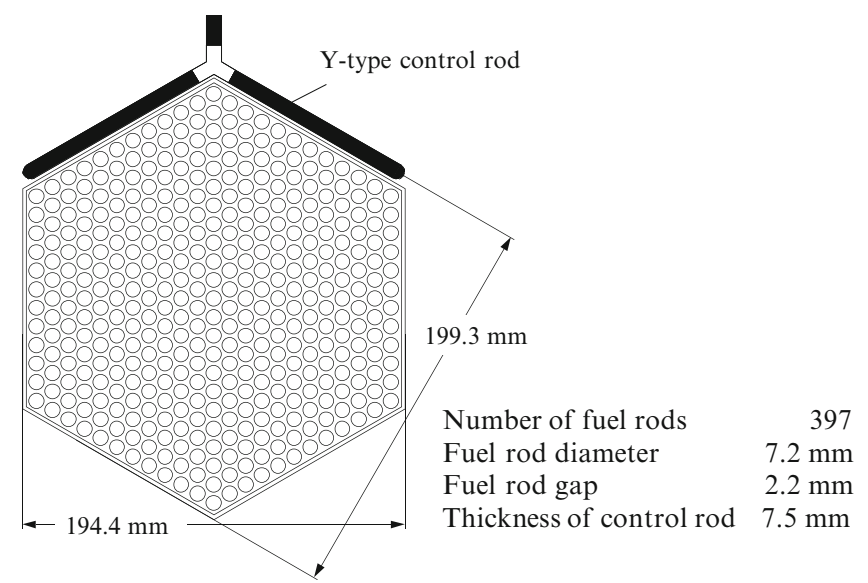

Fig. 14.10 Horizontal cross-sectional view showing configuration of the RBWR-TB2 fuel bundle [3]

with an inner width of $189.6 \mathrm{~mm}$ and wall thickness of $2 \mathrm{~mm}$. The control rod is $7.5 \mathrm{~mm}$ thick, and the gap between the rod outer surface and the channel box is $1.6 \mathrm{~mm}$ on each side. The gap between channel boxes on the side without the control rod is $0.8 \mathrm{~mm}$.

The fuel rod diameter and gap of the RBWR-TB2 are 7.2 and $2.2 \mathrm{~mm}$, respectively; these values result in a larger moderator-to-fuel ratio and a softer neutron energy spectrum than those of the RBWR-TB. Because the fissile composition of the RBWR-TB2 is larger than that of the RBWR-TB, fissile TRUs need to be fissioned with a relatively larger rate to preserve TRU isotopic composition for multi-recycling. The number of fuel rods is the same as that of the RBWR-TB to make the averaged linear heat-generating rate almost the same.

The main core specifications and performance values of the RBWR-TB2 in the equilibrium core were shown earlier in Table 14.2. The core coolant flow is $2.4 \times 10^{4} \mathrm{t} / \mathrm{h}$ at a subcooling of $10 \mathrm{~K}$ at the entrance and has a steam quality of $36 \%$ at the core exit. The concept of the loading pattern of fuel bundles in the equilibrium core is the same as that of the RBWR-TB: it adopts zone loading and the reflective boundary condition of $60^{\circ}$ in the azimuthal direction. A maximum linear heat generation rate of $47 \mathrm{~kW} / \mathrm{m}$ and an MCPR of 1.28 after the control rod scheduling are achieved. The RBWR-TB2 has a void reactivity coefficient of $-4 \times 10^{-4} \Delta \mathrm{k} / \mathrm{k} / \%$ void.

The fission efficiency of TRUs in the RBWR-TB2 is $45 \%$. This value corresponds to about twice the production efficiency of TRUs, $22 \%$, in the ABWR. Here, the production efficiency of TRUs is defined with the opposite meaning of the fission efficiency of TRUs, that is, the net increase in TRUs divided by the total amount of fissioned actinides through the total fuel residence time in the core. As the electricity output of the RBWR-TB2 is the same as that of the ABWR, this means accumulation of TRUs would be suppressed by introducing one RBWR-TB2 for two ABWRs. 
Table 14.3 Trans-uranium (TRU) composition and Puf/TRU weight per fuel batch [3]

\begin{tabular}{|c|c|c|c|c|c|c|c|}
\hline \multirow[b]{2}{*}{ Nuclide } & \multicolumn{2}{|c|}{ RBWR-AC } & \multicolumn{2}{|c|}{ RBWR-TB } & \multicolumn{3}{|c|}{ RBWR-TB2 } \\
\hline & Charge & Discharge $^{a}$ & Charge & Discharge $^{\mathrm{a}}$ & Charge & Discharge $^{a}$ & $\begin{array}{l}\text { LWR spent } \\
\text { fuel }\end{array}$ \\
\hline $\mathrm{Np}-237$ & 0.4 & 0.4 & 0.1 & 0.1 & 1.9 & 1.4 & 6.7 \\
\hline $\mathrm{Pu}-238$ & 2.9 & 2.9 & 4.7 & 4.7 & 6.3 & 6.7 & 2.8 \\
\hline $\mathrm{Pu}-239$ & 43.5 & 43.5 & 9.5 & 9.5 & 27.7 & 25.5 & 48.8 \\
\hline $\mathrm{Pu}-240$ & 36.3 & 36.3 & 39.5 & 39.6 & 38.5 & 40.1 & 23 \\
\hline $\mathrm{Pu}-241$ & 5.1 & 5.1 & 4.4 & 4.4 & 5.5 & 5.4 & 7 \\
\hline $\mathrm{Pu}-242$ & 5.1 & 5.1 & 25.4 & 25.4 & 9.6 & 10.1 & 5 \\
\hline Am-241 & 3.6 & 3.6 & 4.7 & 4.7 & 5.4 & 5.4 & 4.7 \\
\hline $\mathrm{Am}-242 \mathrm{~m}$ & 0.2 & 0.2 & 0.2 & 0.2 & 0.2 & 0.2 & 0 \\
\hline Am-243 & 1.3 & 1.3 & 4.7 & 4.7 & 2.4 & 2.4 & 1.5 \\
\hline $\mathrm{Cm}-244$ & 1.1 & 1.1 & 4.1 & 4 & 1.8 & 2 & 0.5 \\
\hline $\mathrm{Cm}-245$ & 0.4 & 0.4 & 1.2 & 1.2 & 0.5 & 0.6 & 0 \\
\hline $\mathrm{Cm}-246$ & 0.1 & 0.1 & 1 & 1 & 0.2 & 0.2 & 0 \\
\hline $\mathrm{Cm}-247$ & 0 & 0 & 0.2 & 0.2 & 0 & 0 & 0 \\
\hline $\mathrm{Cm}-248$ & 0 & 0 & 0.2 & 0.2 & 0 & 0 & 0 \\
\hline $\mathrm{Cm}-249$ & 0 & 0 & 0.1 & 0.1 & 0 & 0 & 0 \\
\hline Puf(t) & 1.94 & 1.96 & 1.14 & 1.06 & 2.06 & 1.74 & 0.32 \\
\hline TRU(t) & 3.99 & 4.03 & 8.18 & 7.62 & 6.20 & 5.63 & 0.58 \\
\hline
\end{tabular}

${ }^{\mathrm{a}}$ Three-year cooling time after discharge was assumed

Table 14.3 summarizes TRU compositions and weights of fissile plutonium and TRU of charged and discharged fuels in the RBWR-AC, -TB, and -TB2. In evaluation of the discharged fuel compositions, a 3-year cooling time after discharge from the core is considered. Because the RBWR-AC and -TB satisfy the multi-recycling criteria under the condition that both reactors charge TRUs that were discharged from themselves, the TRU compositions of the RBWR-AC and -TB are kept the same in the charged and discharged fuels. The weights of fissile plutonium and TRU increase slightly in the discharged fuel in the RBWR-AC, the break-even reactor, whereas they decrease in the discharged fuel in the RBWR-TB, the TRU burner. As TRUs from LWR spent fuels are added to TRUs discharged from the RBWR-TB2 itself, the TRU composition of their mixture is to be the same at every operation cycle with the constant mixing ratio of TRUs discharged from the RBWR-TB2 and LWR. The weight of TRU decreases in the discharged fuel in the RBWR-TB2.

\subsection{Conclusion}

The specific design and core characteristics of the RBWR were summarized from a review of published studies. The RBWR is categorized as a low moderation LWR. By utilizing a tight lattice fuel and two-phase flow of coolant, the latter of which is a 
feature of BWRs, the moderator-to-fuel ratio of the RBWR can be reduced to a small enough value as to achieve multi-recycling of TRUs.

Different RBWR cores have been designed for different purposes. The RBWR$\mathrm{AC}$ is a break-even reactor with a Pu breeding ratio more than 1.0. The RBWR-TB and RBWR-TB2 are TRU burners that can fission TRUs at a rate more than twice the rate of TRU production by the ABWR. Each of the reactor types achieves the foregoing performances under the condition requiring negative void reactivity coefficient and multi-recycling capability. With the multi-recycling capability, the RBWR-AC/TB/TB2 can continue to fission or recycle TRUs while maintaining the criticality and fulfilling the various constraints, such as sufficient core shutdown margin and negative reactivity coefficient.

The RBWR appears to be a promising candidate energy source that responds to the needs for energy security, for reducing greenhouse-gas emissions, and for mitigating the negative environmental impact of TRUs.

Open Access This chapter is distributed under the terms of the Creative Commons Attribution Noncommercial License, which permits any noncommercial use, distribution, and reproduction in any medium, provided the original author(s) and source are credited.

\section{References}

1. Takeda R, Miwa J, Moriya K (2007) BWRs for long-term energy supply and for fissioning almost all transuraniums. In: Proceedings of the GLOBAL 2007, Boise, ID, September 9-13

2. Takeda R, Miwa J, Moriya K (2012) RBWRs for fissioning almost all uranium and transuraniums. Trans Am Nucl Soc 107:853

3. Hino T, Ohtsuka M, Takeda R, Miwa J, Moriya K (2014) Core designs of RBWR (resourcerenewable BWR) for recycling and transmutation of transuranium elements: an overview. In: Proceedings of ICAPP 2014, Charlotte, April 2014

4. Ducat GA et al (1974) Evaluation of the Parfait blanket concept for fast breeder reactors. MITNE-157. http://inis.iaea.org/search/search.aspx?orig_q=RN:5141813

5. Morimoto Y, Maruyama H, Ishii K, Aoyama M (1989) Neutronic analysis code for fuel assembly using a vectorized Monte Carlo method. Nucl Sci Eng 103:351-358

6. Downar T, Hall A, Jabbay D, Ward A, Greenspan E, Ganda F, Bartoloni F, Bergmann R, Varela C, Disanzo C, Kazimi M, Karahan A, Shwageraus E, Feng B, Herman B (2012) Technical evaluation of the Hitachi Resource-Renewable BWR (RBWR) design concept. EPRI Technical Report 1025086

7. Yang WS, Kim TK, Grandy C, Hill RN (2008) Performance characteristics of metal and oxide fuel cores for a $1000 \mathrm{MWt}$ advanced burner reactor. In: Proceedings of ARWIF08, Fukui, February 2008 